\title{
LA SOCIEDAD COLOMBIANA DE MASTOZOOLOGÍA Y SU PAPEL EN EL DESARROLLO DE LA CIENCIA MASTOZOOLÓGICA EN COLOMBIA
}

\author{
JOSÉ F. GONZÁLEZ-MAYA ${ }^{1,2,4}$ Y HUGO MANTILLA-MELUK ${ }^{1,3}$ \\ ${ }^{1}$ Sociedad Colombiana de Mastozoología, SCMas, Bogotá, Colombia. \\ ${ }^{2}$ Proyecto de Conservación de Aguas y Tierras - ProCAT Colombia, Calle 15 No. 5-52, El \\ Rodadero, Santa Marta, Colombia. \\ ${ }^{3}$ Department of Biological Sciences, Texas Tech University, Main and Flint, Lubbock, TX \\ 79409 \\ ${ }^{4}$ Laboratorio de ecología y conservación de fauna silvestre. \\ Instituto de Ecología, Universidad Nacional Autónoma de México, Ciudad Universitaria, \\ México D.F., México. \\ correo electrónico:jfgonzalez@procat-conservation.org; hugo.mantilla@ttu.edu
}

\begin{abstract}
En noviembre de 2010 se creó la Sociedad Colombiana de Mastozoología, SCMas, en el marco del III Congreso Colombiano de Zoología, un evento de gran trascendencia para el desarrollo de la ciencia de la mastozoología en el país. La SCMas viene a culminar un largo proceso de desarrollo e historia de esta ciencia en Colombia al reunir en un mismo espacio a los investigadores, estudiantes y en general interesados en el estudio de los mamíferos del país. La sociedad busca apoyar y estimular el desarrollo de esta ciencia en Colombia y en la región, promoviendo la investigación y conservación de calidad en el país, facilitando los espacios, interactuando con los actores legales y académicos en favor de un adecuado ejercicio mastozoológico y facilitando espacios para la discusión, crecimiento y difusión de resultados.
\end{abstract}

Key words: Mammalogy, Colombia, Society, Mammals, South America.

Palabras clave: Mastozoología, Colombia, Sociedad, Mamíferos, Suramérica.

Durante el III Congreso Colombiano de Zoología, realizado en noviembre de 2010 en Medellín, Colombia, se desarrolló la primera reunión de la Red Latinoamericana de Mastozoología (RELAM), en su capítulo de Colombia, con la finalidad de establecer la Sociedad Colombiana de Mastozoología, SCMas. La iniciativa para la creación de la SCMas representa un avance fundamental en el desarrollo de la actividad mastozoológica en el país
(González-Maya, 2011) y es identificada por la comunidad de mastozoólogos de Colombia como un capítulo consecuente e hilado a una larga historia de actividad profesional en esta área del conocimiento en el país (Mantilla-Meluk, 2010).

Colombia ha sido identificado como centro de alta diversidad de mamíferos en el contexto regional y mundial (Alberico et al., 2000). Esta diversidad ha cobrado un papel protagónico en las diferentes 
actividades y procesos culturales y académicos de la nación, hasta llegar a ser la actividad de orientación científica de la actualidad, con un importante número de científicos dedicados a este campo y recibiendo una alta atención desde el punto de vista político, social y cultural. Desde esta perspectiva, la diversidad mastozoológica históricamente constituyó un elemento fundamental de la cosmogonía de los primeros pueblos asentados en el territorio colombiano. Estos colonizadores nos legaron muestras de infinita belleza de su interpretación de la diversidad mastozoológica, no sólo en la pintura rupestre, estatuaria y orfebrería precolombinas, sino también en el legado lingüístico, salvaguardado en los dialectos de los más de 81 grupos indígenas colombianos aún existentes (Rodríguez-Mahecha et al., 1995). Como actividad científica, la mastozoología en Colombia tiene sus orígenes en las ideas enciclopedistas de la ilustración del siglo XIX, que resultaron en los primeros intentos de anotaciones ordenadas sobre los mamíferos del territorio nacional escritas por naturalistas como Alexander von Humboldt y Aimé Bonpland (Humboldt y Bonpland, 1812), quienes visitaron el país en este período motivados por la documentación de las riquezas de las colonias de ultramar. Este ejemplo fue rápidamente imitado por naturalistas locales (De Mosquera, 1813) marcando así el punto de partida de una aventura de conocimiento de las especies de mamíferos que con los años ha ido ganando en estructura, la mastozoología moderna en Colombia.
Pueden sugerirse tres períodos básicos en el desarrollo de la mastozoología moderna en Colombia: Inicio, marcado por las primeras colectas con fines museológicos realizadas entre los finales del siglo XIX y principios del siglo XX por colectores profesionales pagados por instituciones americanas $y$ europeas, quienes organizaron expediciones de gran envergadura tanto en su duración (décadas en algunos casos), como en su cobertura geográfica a lo largo del territorio colombiano. A este período le sigue el que podría ser identificado como el Establecimiento a lo largo de la primera mitad del siglo XX, que se caracteriza por la fundación de las primeras colecciones científicas en instituciones académicas. Ejemplos de éstas son las colecciones del Colegio Mayor de San José y el Colegio de la Salle iniciadas por los hermanos Apolinar y Nicéforo María, la colección científica de la Universidad del Cauca que fundó Carlos Federico Lehman y la colección del Instituto de Ciencias Naturales de la Universidad Nacional de Colombia emprendida por Jorge Ignacio HernándezCamacho (Mantilla-Meluk, 2010). La Consolidación de la mastozoología colombiana estaría marcada por la llegada de mastozoólogos profesionales al país que establecen una serie de estudios de carácter sistemático de apoyo a la investigación de las zoonosis tropicales relacionadas con las especies de mamíferos en la década de los 1960’s. La escuela de la Universidad de Los Andes y la de la Universidad del Valle, en conjunto con el Instituto Nacional de Salud, inician 
sus colecciones científicas con énfasis en los mamíferos en asociación con centros de estudios de enfermedades tropicales (Mantilla-Meluk, 2010). A esta etapa se asocian los nombres de R. Tamsitt, D. Valdivieso, M. Marinkelle, R. B. Mackenzey y S. Rengifo.

En la etapa de consolidación, la mastozoología como actividad académica en Colombia es iniciada por J. I. Hernández-Camacho quien contribuye al entrenamiento científico de posteriores protagonistas de la mastozoología colombiana como J. V. RodríguezMahecha y E. Barriga. Finalmente, la mastozoología como actividad científica y académica en Colombia en su periodo Moderno se asocia a la consolidación de la cátedra mastozoológica estructurada por Alberto Cadena en la Universidad Nacional de Colombia donde inicia labores de enseñanza en 1968. Este académico enlazó la mastozoología colombiana con la escuela de Joseph Grinnell, al incorporar su formación bajo la dirección de Jhon Knox Jones Jr. en Kansas University y Texas Tech University al proceso de educación superior colombiano. Una década más tarde esta tarea es complementada por Michael Alberico en la escuela de la Universidad del Valle, quién aportó desde la mastozoología que se desarrollaba en la escuela de la Universidad de Nuevo Mexico y quién contribuyó enormemente a esta ciencia del país con la formación de numerosos recursos humanos, representados en más de 25 cohortes de estudiantes, así como más de 50 publicaciones principalmente enfocadas en dilucidar aspectos de la taxonomía y distribución de especies del Pacífico colombiano, lo que le representó a su vez el aprecio y reconocimiento a nivel nacional e internacional. Derivadas de las cohortes de estudiantes de estos dos mastozoólogos, hoy en día en Colombia contamos con firmes escuelas de mastozoología en todo el territorio nacional, a las cuales se han sumado las incorporaciones de investigadores nacionales y extranjeros que han llegado, o regresado, atraídos por la riqueza mastozoológica del país.

Los estudiantes y profesionales de la biología que se identifican como mastozoólogos en Colombia probablemente se cuentan por centenares. Es así que como respuesta natural a la necesidad de organización de un volumen creciente de profesionales en el área, emerge la Sociedad Colombiana de Mastozoología (SCMas) que encontró su punto de catálisis y motor de acción en la creación de la RELAM durante el X International Mammalogical Congress, en Mendoza, Argentina, en el 2009 (MantillaMeluk, 2009). La creación de la Sociedad representa una oportunidad única para estimular, apoyar y desarrollar la ciencia alrededor del estudio de los mamíferos en el país.

En años recientes, el estudio mastozoológico en Colombia ha venido en incremento gracias a la formación de nuevos investigadores, el aumento en el flujo de científicos nacionales a realizar estudios de posgrado en el exterior, y a la atención y facilidades que ha recibido esta ciencia y que está aumentando en 
términos generales en el país. Este incremento ha sido evidenciado a través del aumento significativo en el número de publicaciones referentes al tema en revistas nacionales e internacionales, y al aumento de proyectos relacionados con mastozoología en todo el territorio nacional. Sin embargo, aún hace falta un mayor estímulo para los estudiantes de pregrado y posgrado para desarrollar sus trabajos de tesis en temas mastozoológicos. Pero sobre todo, difundir estos resultados es fundamental, de forma que se vaya conglomerando un conocimiento profundo y completo de este grupo taxonómico en el país y que sea accesible más allá de los anaqueles de las universidades.

La SCMas viene a llenar este importante vacío proporcionando los espacios y estímulos necesarios para el desarrollo de la mastozoología colombiana, valiéndose de múltiples medios para lograr sus objetivos. Para este fin, la SCMas se plantea como objetivos: a) fomentar y promover la coordinación interinstitucional y la integración interdisciplinaria de los estudios sobre los mamíferos a nivel nacional y regional; b) promover y contribuir al desarrollo de una formación teórica moderna e integral en los profesionales y los investigadores jóvenes Colombianos, estableciendo los vínculos necesarios con las sociedades e instituciones académicas y civiles acreditadas que promuevan el estudio de los mamíferos en el mundo; c) representar a la actividad científica Colombiana en el estudio de los mamíferos y a sus profesionales, ante las instituciones públicas y privadas nacionales e internacionales $\mathrm{y}$, en particular, ante los organismos y asociaciones dedicadas al estudio de los mamíferos de otros países; d) promover y realizar actividades culturales y académicas de extensión y divulgación en diversos aspectos de las investigaciones sobre mamíferos, en particular mediante publicaciones, reuniones científicas periódicas, conferencias, mesas redondas, talleres y cursos de postgrado; e) contribuir a resolver los problemas que atañen a los académicos dedicados al estudio de los mamíferos a nivel nacional, en especial aquellos de carácter operativo (concentración, activación y utilización de las colecciones, destino de ejemplares tipo, acceso a la bibliografía, financiamiento de viajes de estudio y participación en congresos, maximización del aprovechamiento de los resultados de las colectas y trabajos de campo, facilitación de la publicación de las investigaciones concluidas); f) contribuir a preservar la fauna nativa de mamíferos vivientes, sus ambientes naturales y los yacimientos de mamíferos fósiles, promoviendo acciones para que se respete la legislación reguladora y de conservación existente y promoviendo, en caso necesario, legislaciones complementarias; g) defender los intereses nacionales y contribuir a regular las formas de participación extranjera en el estudio de los mamíferos de la fauna de Colombia, viviente y extinta, promoviendo la orientación de la investigación internacional sobre nuestros mamíferos en beneficio de las colecciones y los 
centros de investigación nacionales, y de la formación y entrenamiento de los jóvenes científicos Colombianos; h) efectuar una publicación con característica de revista de aparición regular sobre temas mastozoológicos, comprendiendo los aspectos científicos, culturales, académicos, de integración interdisciplinaria y de carácter intra- e interinstitucional que contribuya a los objetivos de la asociación; e i) realizar todo tipo de acción o actividad organizativa, cultural y académica no prevista en la enumeración anterior, pero que sea acorde con los objetivos señalados.

Para lograr sus objetivos, la SCMas ha iniciado sus labores con la realización del I Congreso Colombiano de Mastozoología en conjunto con la Universidad Tecnológica del Chocó, Diego Luis Córdoba, celebrado los días 19 al 23 de septiembre de 2011 en la ciudad de Quibdó, Chocó. Este evento tuvo como uno de sus ejes la consolidación de la Confederación de Sociedades Mastozoológicas del Chocó Biogeográfico con las naciones hermanas de Ecuador y Panamá. En la actualidad la SCMas además se encuentra editando el primer volumen del Boletín de la Sociedad Colombiana de Mastozoología, y participa activamente de la Red Latinoamericana de Mastozoología y de la Federación Internacional de Mastozoólogos. Por último es importante mencionar que esta Federación ha avalado la SCMas como la decimoséptima sociedad oficial de mastozoología a nivel global.
Los invitamos a hacerse participes de ésta iniciativa y aportar al crecimiento de la sociedad que esperamos rinda grandes frutos en el futuro próximo y represente un gran avance en el estudio, entendimiento y conservación de la rica mastofauna del país.

\section{AGRADECIMIENTOS}

Agradecemos enormemente a los participantes de la primera reunión realizada durante el IIICCZ en Medellín, y especialmente a los miembros de su junta directiva, quiénes voluntariamente han venido aportando al desarrollo y construcción de la sociedad: Adriana Ruiz, Danny Zurc, Miguel Eduardo Rodríguez, Alberto Cadena, Ivan Mauricio Vela, Alex Mauricio JiménezOrtega, Gabriel Pantoja, Edgar Daniel Rodríguez, Andrés Quintero Angel y Jesús Ballesteros. Por último, a los editores y revisores de la RMM y en especial al Dr. Alberto Cadena por los valiosos aportes que mejoraron sustancialmente este manuscrito.

\section{LITERATURACITADA}

Alberico, M.A., A. Cadena García, J.I. Hernández-Camacho y Y. Muñoz-Saba .2000. Mamíferos (Synapsida: Theria) de Colombia.Biota Colombiana, 1: 43-75.

De Mosquera, T.C. 1853. La Nueva Granada. Physical and political geography of Nueva Granada.The Geographical and Statistical Society of New York,1-105.

González-Maya, J.F. 2011. Creación de la Sociedad Colombiana de Mastozoología. Boletín Alúna, 3(2):20. 
Humboldt, A. von y A. Bonpland. 1812. Recueil d' observations de zoologie et d' anatomie comparée, faites dans l'ocean atlantique dans l'interieur du nouveau continent et dans la mer du sud pendant le années 1799, 1800, 1801, 1802 et 1803. pt. 2, vol. 1, Paris, viii + 368pp.

Mantilla-Meluk, H. 2009. Sobre la importancia del ICM 10 (International Mammalogical Congress) para la comunidad mastozoológica latinoamericana. Mastozoología Neotropical, 16(2):287-289.

Mantilla-Meluk, H. 2010. Sobre la conformación de la Sociedad Colombiana de Mastozoología (SCMas). Mastozoología Neotropical, 17(2):257-261. 